\title{
The lung microbiome in neonates
}

\author{
Dhivya Lakshmi Permall ${ }^{1}$, Asfia Banu Pasha ${ }^{1}$, Xiao-qing Chen ${ }^{1}$, Hong-yan $\mathrm{Lu}^{2}$ \\ ${ }^{1}$ Department of Pediatrics, The First Affiliated Hospital of Nanjing Medical University, No 300, Guangzhou Road, \\ Nanjing, Jiangsu Province; ${ }^{2}$ Department of Pediatrics, Affiliated Hospital of Jiangsu University, Zhenjiang, China. E-mail: \\ zysktz@163.comorlhy5154@163.com \\ Received: 10th December 2018, Revised: 1st May 2019, Accepted: 25th July 2019
}

SUMMARY: Permall DL, Pasha AB, Chen XQ, Lu HY. The lung microbiome in neonates. Turk J Pediatr 2019; 61: 821-830.

Despite the advent of culture-independent techniques to identify members of the microbiome, studies focusing on the lung microbiome of neonates are scarce. Understanding the role of the microbiome in the pathogenesis of pulmonary conditions affecting newborns could lead to the initiation of pioneering therapeutic interventions, which could potentially prevent lifelong disability. Bronchopulmonary dysplasia (BPD) has been associated with a less diverse microbiome, presence of Ureaplasma species and reduced Lactobacillus detection. Additionally, the potential role of microbial dysbiosis in the pathogenesis of asthma, cystic fibrosis and pneumonia has been described. There has also been a surge of interest in attempting to elucidate the interactions between the airway and gut microbiomes and their bearings on respiratory health and diseases to eventually broaden the scope of therapeutic interventions.

Key words: airway, bronchopulmonary dysplasia, lung, microbiome, newborn.

Deciphering the lung microbiome in health and disease is an emerging area of interest. Identification of bacteria through the revolutionary culture-independent methods by sequencing the variable regions of the 16S ribosomal RNA gene, has defied the previous notion of airway sterility. ${ }^{1,2}$ The airway microbiome harbors niche-specific microbial communities and potentially acts as a gatekeeper, protecting the respiratory tract from colonization by harmful pathogens. ${ }^{3}$ The respiratory tract is in constant contact with the external environment and the microbial load it harbors is higher in the upper airways and significantly reduced in the lower airways. ${ }^{4}$ Apart from ethical and practical difficulties surrounding clinical studies involving newborns, sampling difficulty, contamination and low bacterial biomass are added challenges to surmount. ${ }^{5}$ This review focuses on the newborn respiratory microbiome and its potential causality in pulmonary diseases in neonates.
Factors influencing the lung microbiome in newborns

Being a dynamic ecological system, the composition of the lung microbiome is determined by 3 key factors: immigration, elimination and reproduction rates of the various constituents of the microbiome. ${ }^{6}$ These factors have an incessant impact on the lung microbiome. The main mechanism of immigration is through microaspiration whereas the elimination process involves cough reflex, mucociliary clearance and immune responses. ${ }^{6,7}$ Reproduction rates of the members of the microbiome depend on the characteristics of the lung's interior milieu, namely partial pressure of oxygen, $\mathrm{pH}$, blood supply, alveolar ventilation, temperature and inflammatory cells availability and response. ${ }^{6}$ In healthy conditions, the microbiome is largely determined by immigration and elimination and in diseased condition, by regional growth conditions. ${ }^{6,8}$ Except for the above mentioned main determinants of the lung microbiome, several other factors have been reported to influence it. 


\section{Delivery mode}

Previously, a significant relationship between earliest microbiome of the newborn and delivery mode had been reported. ${ }^{9}$ Newborns delivered vaginally displayed bacterial species correlating with maternal vaginal flora, mainly Lactobacillus, Prevotella, or Sneathia species and those delivered via cesarean section presented with maternal skin bacterial communities, the majority being Staphylococcus, Corynebacterium, and Propionibacterium species. ${ }^{9}$ However, another study found no significant differences between the lung microbiome of extremely low birth weight (ELBW) infants born either vaginally or by cesarean section. ${ }^{10}$ Interestingly, a potential explanation for this finding might be related to the existence of a placental microbiome, as described by Aagaard et al. ${ }^{11}$ The amniotic fluid has also been found to be contaminated with diverse microbes, although their roles have yet to be clarified. ${ }^{12}$ The process of microbiome establishment might thus begin antenatally.

\section{Feeding method}

It is common knowledge that breastfeeding is the most favorable feeding method for newborns. Consistently, a higher percentage of 6-week old breastfed infants compared to formula-fed ones were found to harbor Corynebacterium and Dolosigranulum in the nasopharynx and the latter has been found to confer protection against wheezing and mild respiratory tract infections in less than 6-months old infants. ${ }^{13}$ Transfer of protective maternal antibodies and valuable bacterial species, namely Bifidobacterium and Lactobacillus, are only possible through breastfeeding. ${ }^{3}$ The presumed optimal microbiome of newborns is achieved through the combination of vaginal delivery and consequent exclusive breastfeeding. ${ }^{5}$

\section{Exposure to chorioamnionitis}

Chorioamnionitis is one of the risk factors for preterm labor and it can be diagnosed prenatally by maternal findings or postnatally by histological evaluation of the placenta. ${ }^{14}$ The causative organisms are thought to potentially originate from the maternal vaginal flora. ${ }^{15}$ The respiratory microbiome of ELBW infants exposed to chorioamnionitis was observed to have decreased genus Lactobacillus, which has shown an association with subsequent BPD development. ${ }^{10}$ Moreover, the finding of a less diverse microbiome in the tracheal aspirates of preterm infants with a history of chorioamnionitis exposure might be a sign of increased pathogenic bacteria community. ${ }^{5,16}$

\section{Antibiotic use}

Antibiotics have been reported to interfere with gut and respiratory microbiomes, leading to less diversity and reduction in potentially favorable commensal bacteria. ${ }^{3,5}$ By altering the presumed unstable intestinal microecology of newborns, antibiotic administration can modify the host immune responses to allergens, with subsequent asthma development as one of the negative outcomes. ${ }^{17,18}$ Antibioticinduced early intestinal dysbiosis has been associated with greater probability of early asthma development and may lead to persistent infection. ${ }^{19,20}$ Varon et al. ${ }^{21}$ assessed the presence of Streptococcus pneumoniae, Haemophilus influenzae, and Moraxella catarrhalis in the nasopharynx of infants with respiratory tract infections before and after antibiotic treatment. The study revealed a significant reduction in the proportion of the three species, with an increase in the proportion of penicillin-resistant Streptococcus pneumoniae due to a decrease in the proportion of penicillinsensitive Streptococcus pneumonia. Moreover, a longitudinal study by Salter et al..$^{22}$ found that the impact of respiratory diseases and antibiotic use on the nasopharyngeal mibrobiota was a disordered microbial environment, which differed across individuals and diseases. However, prophylactic administration of antibiotics to mothers before preterm delivery does not influence the respiratory mibrobiome composition of premature newborns. ${ }^{10}$

\section{Relationship between lung microbiome and intestinal microecology}

The microbiome of the gastrointestinal tract is the most populated one with an estimated $10^{14}$ bacteria whereas the lower airway microbiome harbors only approximately 10-100 bacteria per 1,000 human cells. ${ }^{17}$ Having the same embryonic origin, the gut and the respiratory 
tract share structural resemblances, despite dissimilar environmental characteristics and functions. ${ }^{23}$ Extensive study of the gut microecology has been feasible due to the ease of fecal sample collection and the abundant microbial community members residing in the gut. ${ }^{23}$ However, the more challenging study of respiratory microbiome faces numerous obstacles such as the low microbial load of the lung, bronchoscopic contamination upon sample retrieval, continuous dissemination from oral or gastrointestinal sites and elimination mechanisms. ${ }^{23}$ The role of microaspiration in establishing the airway microbiome is illustrated by the presence of microbes, originally from the intestinal microecology, in the respiratory tract. ${ }^{17}$ The intestinal microecology is lead by the main four bacterial phyla, namely, Firmicutes, Bacteroidetes, Proteobacteria and Actinobacteria but the major phyla in both gut and lung microenvironments are Bacteroidetes and Firmicutes. ${ }^{17,23}$ The term gut-lung axis is used given the modulating effect on the gut microbiome on the lung. For example, Arrieta et al. ${ }^{19}$ observed a temporary intestinal microbial dysbiosis in the first 100 days of life in infants at greater risk of asthma, with recovery of lost microbial members in murine models leading to less airway inflammation. Additionally, intestinal microbial diversity investigations revealed increased asthma diagnoses in children with lower microbial diversity at 1 week and 1 month of age, emphasizing the magnitude of factors influencing the microbiome early in life. ${ }^{24}$ The neonatal period is a decisive time for the development of the immune system that has a lifelong impact on an individual's health. ${ }^{18}$ Notably, the presence of favorable microbes such as Bifidobacterium longum is associated with decreased prevalence of asthma. ${ }^{18}$ Short chain fatty acids (SCFA) produced by the gut microbes can also influence the fate of respiratory conditions. ${ }^{25}$ As demonstrated by Trompette et al. ${ }^{26}$ in their study involving mice, a higher proportion of circulating SCFA can confer a protective effect against allergic disease development. Chen et al. ${ }^{27}$ were the first ones to report an inverse relationship between Helicobacter pylori and development of childhood asthma and this finding may be explained by the hygiene hypothesis. ${ }^{28}$ Interestingly, this protective effect of Helicobacter pylori may lead to generation of Helicobacter pylori-based therapeutic compounds to prevent asthma development or its progression. ${ }^{28}$ Furthermore, daily intake of Lactobacillus acidophilus and Bifidobacterium animalis in young children aged 3-5 years decreases the incidence of cold symptoms. ${ }^{29}$ Since intestinal inflammation is a characteristic feature of cystic fibrosis (CF), it exposes the involvement of the gastrointestinal tract in another chronic lung disease. In their study involving CF children, Bruzzese et al. ${ }^{30}$ found a disordered intestinal microbiome in CF patients, aggravated by antibiotic use and partially restored to a healthy status with Lactobacillus GG utilization. Thus, manipulation of the gut microbiota can alleviate pulmonary symptoms or even prevent the occurrence pulmonary diseases. Deeper insight into the novel concept of gut-lung axis is key for the evolution of modern and gut-targeted therapy in the context of pulmonary diseases.

\section{Lung microbiome and pulmonary diseases in neonates}

\section{Bronchopulmonary Dysplasia (BPD)}

Prevention of BPD remains a challenging task in the Neonatal Intensive Care Unit (NICU). Despite advances in non-invasive ventilation strategies and improved survival rates of extremely preterm infants, the BPD incidence has remained stable. ${ }^{31}$ The lung microbiome is now being thoroughly investigated, in search of potential causal factors involved in the pathogenesis of BPD. The existence of Ureaplasma species in the airway of premature infants has been linked to inflammation in the lungs, leading to extended use of supplemental oxygen and thus an augmented BPD risk. ${ }^{32,33}$ This possible relationship has prompted the use of macrolides to target Ureaplasma infection in preterm lungs. Clarithromycin has demonstrated positive outcomes, with a reduction in BPD rates in preterm infants colonized with Ureaplasma urealyticum. ${ }^{34}$ Additionally, azithromycin is a promising therapy but requires further evaluation. ${ }^{35}$ One study found the presence of Corynebacterium species to be more likely in infants with longer duration of mechanical ventilation and severe BPD. ${ }^{36}$ Inflammatory insult might also 
explain this finding; however, additional and larger studies are warranted to recognize the effect of Corynebacterium species on the airway of extremely preterm infants. Wagner et al. ${ }^{33}$ found that development of severe BPD in mechanically-ventilated premature neonates correlated with increased microbial community turnover with time and greater relative abundance of Ureaplasma at birth compared to Staphylococcus. Yet, the association between rate of microbial turnover and BPD severity is still uncertain since the impact of factors such as antibiotic use on the lung microbiome is still imprecise. Potentially owing to hospital environmental differences, the most prevalent organisms in the study by Wagner et al..$^{33}$ were Staphylococcus and Ureaplasma, unlike the report by Lohmann et al. ${ }^{16}$, in which Acinetobacter was dominant. According to the Shannon diversity index, Lohmann et al. ${ }^{16}$ reported a probable connection between an early, initial, less diverse microbiome and subsequent BPD. Nevertheless, lower birth weight and gestational age still significantly influenced BPD incidence. A recent systematic review of 6 studies regarding airway microbiome and BPD occurrence demonstrated: (1) the existence of a respiratory tract microbiome soon after birth, with dominating species being Staphylococcus and Ureaplasma, (2) the relationship between BPD and great airway microbial community, (3) the relationship between BPD and varying quantity of Proteobacteria and Firmicutes and lastly, (4) the link between BPD and lower detection of Lactobacillus. ${ }^{31}$ The role of microbial disruptions in BPD progression is still vague and further research is required to elucidate this relationship.

\section{Asthma}

Asthma is a chronic condition, characterized by airway inflammation, which affects $10 \%$ of the pediatric population. ${ }^{37}$ Microbialhost interactions and modulation of the immune system are closely linked and arising disturbances in any of these factors can cause individuals to be more prone to developing atopic allergic diseases. ${ }^{38}$ Microbiome dysbiosis in the childhood period has been implicated in the pathogenesis of asthma. ${ }^{39}$ Perez et al. $^{40}$ noted a predominance of Proteobacteria compared to Firmicutes from the nasopharyngeal microbiome of preterm infants.
Bisgaard et al. ${ }^{41}$ found that in the neonatal period, hypopharyngeal colonization could be utilized as a potential indicator of early asthma development. Hypopharyngeal colonization with Streptococcus pneumoniae, Haemophilus influenzae, Moraxellacatarrhalis, or a combination of these bacteria, significantly increases the risk of wheezing events, and also leads to a rise in eosinophil and total IgE levels. ${ }^{41}$ Teo et al. ${ }^{42}$ studied the nasopharyngeal microbiome of infants and reported the association of Streptococcus pneumoniae, Haemophilus influenzae and Moraxella catarrhalis with acute respiratory illness and found Streptococcus pneumoniae colonization to be related to consequential chronic wheeze in children who were atopic by the second year of life. In addition, another study in young children found a significant relationship between wheezy episodes and Haemophilus influenzae and Moraxella catarrhalis, but on the other hand, Streptococcus pneumoniae was not found to have a significant association with recurrent wheeze. ${ }^{43}$ The PARSIFAL and GABRIELA studies revealed the protective effect of childhood exposure to multiple and diverse microbial communities against asthma. $4^{4}$ Exposure to a wide array of microorganisms in the farming environment can lead to activation of Toll-like receptors, with subsequent set-off of their respective signaling pathways and a resulting increase in activation of type 1 helper T cells. ${ }^{44}$ This can lead to reduced action of the type 2 helper $\mathrm{T}$ cell, which is distinctively found in asthma. ${ }^{44}$ Using bacteria-free and specific pathogen-free mice, Herbst et al. ${ }^{45}$ also exposed the worth of the microbiome as a shield against asthma.

\section{Cystic Fibrosis (CF)}

$\mathrm{CF}$ is a multi-organ, autosomal recessive condition which occurs due to a defect in the cystic fibrosis transmembrane conductance regulator gene, with involvement of both gram-negative and gram-positive bacteria. ${ }^{46,47}$ A study using newborn pigs revealed inhibition of elimination mechanisms of the respiratory tract, consequently triggering the flow of reactions causing inflammation and subsequent changes in the cystic fibrosis lungs. ${ }^{46}$ In their study, Frayman et al. ${ }^{48}$ assessed the lower respiratory tract microbiome of young children with CF using bronchoalveolar lavage samples. Although a diverse lower airway microflora 
is observed in children with CF, microbial diversity decreases with increasing age; yet, this finding does not correlate with a decreased lung function by the age of 6 years. ${ }^{48}$ Gangell et al. ${ }^{49}$ demonstrated that the organisms with stronger inflammatory effects in the lungs of young children with CF were Staphylococcus aureus, Pseudomonas aeruginosa, and Aspergillus species. Another study examined the relation between lung microbiome, pulmonary function and airway inflammation in children with CF using molecular techniques to analyze saliva, oropharyngeal swabs, induced and expectorated sputum. ${ }^{50}$ Analysis of the collected samples revealed a higher relative abundance of Pseudomonas and Staphylococcus in the lower respiratory tract samples compared to the upper respiratory tract ones. Furthermore, Pseudomonas, Staphylococcus and Enterobacteriaceae were associated with increased airway inflammation and a relationship between the upper and lower respiratory tracts' microbial divergence and an increased sputum neutrophil elastase was noted. ${ }^{50}$ An investigation regarding the changes in the nasopharyngeal microbiome of $\mathrm{CF}$ infants from the time of diagnosis until 6 months of age was conducted by Prevaes et al. ${ }^{51}$ For CF subjects, the findings were as such: (1) initial detection of mainly Staphylococcus aureus and relatively lower levels of Corynebacterium and Moraxella, (2) by 3 months of age, Streptococcus mitis was the main organism detected, and (3) antibiotic usage was followed by a reduction of presumably favorable bacteria related to airway microbial stability (Corynebacterium and Dolosigranulum) with increased pathogenic ones (Gram negative Burkholderia and Enterobacteriaceae species); however the control subjects displayed Moraxella species during the first 6 months of life. ${ }^{51}$ Further antibiotic-related studies are necessary to supplement these results, paving the way for targeted antibiotic treatment. For now, antibiotic prophylaxis therapy is the mainly applied. ${ }^{51}$

\section{Pneumonia}

Studies have found that a disordered respiratory microbiome in the neonatal period may have an impact on maturation of the immune system. ${ }^{52}$ Colonization of the respiratory tract of healthy newborns with Streptococcus pneumoniae,
Haemophilus influenzae, or Moraxella catarrhalis is associated with an increased risk of developing pneumonia. ${ }^{52}$ Different microorganisms are associated with development of different types of pneumonia. According to Sakwinska et al. ${ }^{53}$, a high abundance of Moraxella lacunata complex was found in the nasopharyngeal samples of patients with viral pneumonia and it was also observed to be 7 -fold more abundant in viral compared to non-viral pneumonia patients. Additional viral pathogens detected in the nasopharynx of viral pneumonia patients were respiratory syncytial virus, adenovirus, human metapneumovirus and rhinovirus. ${ }^{53}$ On the other hand, the three most common bacterial pathogens detected in the nasopharynx of patients with bacterial pneumonia included Streptococcus pneumoniae, Haemophilus influenzae and Moraxella catarrhalis; however, Haemophilus influenzae was especially abundant in the nasopharyngeal samples of patients with no definite etiology. ${ }^{53}$ Also, the nasopharyngeal microbiome was observed to be richer and more diverse in healthy children compared to pneumonia patients. ${ }^{53}$ In the search for the definite etiologies of ventilator-associated pneumonia (VAP), a frequent complication of mechanical ventilation in neonates, $\mathrm{Lu}$ et al. ${ }^{54}$ prospectively studied the diversity of microbiome in VAP. Newborns suffering from respiratory distress syndrome (RDS) and VAP constituted the experimental group whereas those suffering from RDS but not VAP formed the control group. Within the first day of intubation, no differences were observed in the constituent ratio of any genus between the two study groups. Within 1-3 days of intubation, the results demonstrated a higher ratio of Klebsiella, Acinetobacter and Streptococcus in the VAP group and a lower ratio of Serratia and Achromobacter compared to the control group. This less diverse lower airway microbiome was induced by VAP. Analysis of sputum samples from 3-5 days after intubation revealed lower proportions of Klebsiella, Acinetobacter, Serratia and Achromobacter but higher proportion of Streptococcus in the VAP group compared to the non-VAP group. Given the study outcomes, the authors concluded that the constituent ratios of Klebsiella, Acinetobacter and Streptococcus could be used as early predictors of VAP in the initial 3 days of intubation. ${ }^{54}$ 


\section{Therapeutic interventions targeting the lung microbiome}

The theory that all bacteria are detrimental to human health has been rendered obsolete and the symbiotic relationship between humans and microbes is now well-appreciated. Constituents of the gut microflora have antiinflammatory, anti-oxidizing and pain-relieving properties. They also produce vitamins and help protect the gut from attachment of pathogenic bacteria which can lead to chronic disease. ${ }^{55}$ In an era of increasing antibioticresistance, researches and clinical trials based on the therapeutic usage of the members of the gut microecology on pulmonary diseases are growing in number. With a biomass of up to 1.5 $\mathrm{kg}$ due to the vast microbial load comprising the gut microbiome, it is occasionally termed as "forgotten organ". ${ }^{55}$ Probiotics are defined as living microorganisms, which when consumed in sufficient amounts, benefits the host's health. ${ }^{56}$ The proposed mechanisms of probiotics incorporate direct antimicrobial activity, strengthening the epithelial barrier and modulation of the immune system..$^{57}$ The most frequent species used to enhance immunity are lactic acid bacteria, namely, specific strains of Lactobacillus and Bifidobacterium. ${ }^{58}$ Their boosting effect on the innate immune system improves the phagocytic functions of peripheral blood polymorphonuclear cells and monocytes, and enhances the cytotoxic ability of NK cells. ${ }^{20}$ Additionally, evidence of the positive effect of probiotics on the acquired immune system is shown by rising levels of IgG, IgA, and IgM immunoglobulins after probiotic intake. ${ }^{20}$ In a study assessing prophylactic probiotic usage for the prevention of VAP in children in the high-risk PICU setting, encouraging results were noted as probiotic use led to a $77 \%$ decrease in VAP incidence. ${ }^{59}$ Moreover, administration of Lactobacillus GG compared to placebo to children in day-care centers reduced the risk of developing upper respiratory tract infections. ${ }^{60}$ In the network meta-analysis evaluating probiotics for the prevention of respiratory infections in children and adolescents by Amaral et al. ${ }^{61}$, Lactobacillus casei rhamnosus uniquely demonstrated a superior effect in decreasing respiratory infection rates compared to placebo, while no beneficial outcome resulted from other probiotic strains. This study reinforces the general agreement that evidence is lacking for implementation of single or mixed probiotics usage therapeutically or prophylactically for respiratory infections. Despite the satisfactory results of probiotic use on respiratory tract infections in terms of fewer days of illness, further, large and more powered studies are warranted to establish the optimal strain, dosage and timing of therapy as well as potential long-term consequences. ${ }^{62}$ The impact of probiotics on asthmatics has been investigated in mice by Zhang et al ${ }^{63}$ and they reported the protective effect of Lactobacillus rhamnosus GG against OVA-induced allergic airway inflammation. Chen et al. ${ }^{64}$ observed an advantageous effect of supplemental Lactobacillus gasseri A5 for a 3-month period in asthmatic children (6-12 years), with improvement in lung function and alleviation of asthmatic symptoms. Conversely, a recent meta-analysis found no benefits of probiotic supplementation during pregnancy or early life since no significant reductions in incidence of asthma or wheeze in infants were observed. Accordingly, the use of probiotics for the prevention of asthma in infants is not supported. ${ }^{65}$ In the context of $\mathrm{CF}$, although the results of studies included in recently published systematic reviews endorse the use of probiotics to lower the rate of pulmonary exacerbations, the included studies were underpowered and had several limitations, such as insufficient sample size and short duration of probiotic supplementation, to determine the actual effect of probiotics. ${ }^{66-68}$ Furthermore, as negative results are seldom published, it eventually leads to publication bias in literature analysis. In a recent double blind crossover placebo controlled study, Van Biervliet et al. ${ }^{69}$ could not validate the previously reported beneficial effects of probiotic supplementation since no significant associations were found for fecal calprotectin and pulmonary exacerbations; although a positive effect on gut permeability was noted. Additionally, Bruzzese et al. ${ }^{70}$ designed a multicentre RCT to evaluate the effect of Lactobacillus GG, supplemented for a period of 12 months, on pulmonary exacerbations and hospitalization in children aged 2-16 years 
of age with CF. The results demonstrated no benefit of Lactobacillus GG on respiratory outcomes. Another choice of therapy can be the use of prebiotics, which are indigestible substances used to fuel the growth and effect of specific microbes to eventually improve the host's health. ${ }^{71}$ Conflicting outcomes also burdens the recommendation for the routine use of prebiotics as prophylaxis in at-risk children. A large study found the galacto-oligosaccharide/polydextrose (GOS/PDX)-formula to have a significant protective effect against respiratory infections in young infants. ${ }^{72}$ Yet, another large trial study reported no effect of a GOS-containing infant formula on the rate of infections or allergic diseases in infants, even though a clear prebiotic effect was demonstrated by changes in feacal characteristics. ${ }^{73}$ Moreover, a RCT by Niele et al. ${ }^{74}$, including 113 preterm infants, found no benefits of short-term prebiotic mixture supplementation of neutral and acidic oligosaccharide on the incidence of infectious and allergic conditions in infants during their first year of life. Interestingly, a recent study concluded that prebioticsupplemented formula compared to breast milk in infants was found to have comparable prophylaxis effects for respiratory infections, diarrhea and constipation. ${ }^{75}$ Thus, in the case of breast milk unavailability, prebioticsupplemented formula could be used as a replacement. Nevertheless, whether with the use of prebiotics or probiotics, consideration of host factors is essential as individuals differ from another in terms of genetic background, composition of intestinal mibrobiome, dietary habits and other aspects of living. ${ }^{76}$ Since the maternal microbiome is intimately connected to the infant intestinal microbiome, a prospective substitute approach to reduce the risk of allergic diseases in infants could be the manipulation of maternal microbiome. ${ }^{71}$ Under the circumstance that dysbiosis of the respiratory microbiome is found to be the underlying trigger of a certain disease, there will be a pressing need to evaluate the lung microbiome as a therapeutic target. ${ }^{77}$ Research aimed at assessing the effect of lung microbiome manipulation in restoring the balance within the lung microenvironment will boom.

\section{Summary and Expectations}

In brief, the lung microbiome has now gained the spotlight of research but studies during the critical neonatal period are still lacking. Since BPD is the most debilitating disease affecting premature neonates, depicting the underlying mechanisms of how a disordered airway microbiome influences its pathogenesis could be a potentially groundbreaking revelation for improved prevention and therapeutic interventions. Early exposure to a diverse range of microbes in children has been found to reduce the risk of developing asthma. Additionally, specific pathogens have been detected in a chronological manner in CF patients. "Respiratory probiotics" might be a potential tool to positively influence the microbiome at birth. ${ }^{10}$ Moreover, future studies should aim to identify the viral and fungal communities and the intermicrobial interactions for a more in-depth understanding of the respiratory microbiome and its function in the pathophysiology and evolution of pulmonary diseases. Grasping the machinery of the microbiome will be a step further towards the aim that is individualized medicine.

\section{Funding}

This work was supported by the National Natural Science Foundation of China (No. 81741052, 81871195) and Clinical Research Cultivation Program (2017CX015).

\section{REFERENCES}

1. Lanaspa M, Bassat Q, Medeiros MM, Munoz-Almagro C. Respiratory microbiota and lower respiratory tract disease. Expert Rev Anti Infect Ther 2017; 15: 703711 .

2. Cui L, Morris A, Huang L, et al. The microbiome and the lung. Ann Am Thorac Soc 2014; 11(Suppl 4): S227-S232.

3. Man WH, de Steenhuijsen Piters WA, Bogaert D. The microbiota of the respiratory tract: gatekeeper to respiratory health. Nat Rev Microbiol 2017; 15: 259270.

4. Lynch SV. The lung microbiome and airway disease. Ann Am Thorac Soc 2016; 13(Suppl 5): S462-S465.

5. Gallacher DJ, Kotecha S. Respiratory microbiome of new-born infants. Front Pediatr 2016; 4: 10. 
6. Dickson RP, Erb-Downward JR, Huffnagle GB. Homeostasis and its disruption in the lung microbiome. Am J Physiol Lung Cell Mol Physiol 2015; 309: L1047-L1055.

7. Tracy M, Cogen J, Hoffman LR. The pediatric microbiome and the lung. Curr Opin Pediatr 2015; 27: 348-355.

8. Faner R, Sibila O, Agusti A, et al. The microbiome in respiratory medicine: current challenges and future perspectives. Eur Respir J 2017; 49: 1602086.

9. Dominguez-Bello MG, Costello EK, et al. Delivery mode shapes the acquisition and structure of the initial microbiota across multiple body habitats in newborns. Proc Natl Acad Sci U S A 2010; 107: 11971-11975.

10. Lal CV, Travers $\mathrm{C}$, Aghai $\mathrm{ZH}$, et al. The airway microbiome at birth. Sci Rep 2016; 6: 31023.

11. Aagaard K, Ma J, Antony KM, Ganu R, Petrosino $\mathrm{J}$, Versalovic J. The placenta harbors a unique microbiome. Sci Transl Med 2014; 6: 237ra65.

12. DiGiulio DB. Diversity of microbes in amniotic fluid. Semin Fetal Neonatal Med 2012; 17: 2-11.

13. Biesbroek G, Bosch AA, Wang X, et al. The impact of breastfeeding on nasopharyngeal microbial communities in infants. Am J Respir Crit Care Med 2014; 190: 298-308.

14. Jobe A. Effects of chorioamnionitis on the fetal lung. Clin Perinatol 2012; 39: 441-457.

15. Chu DM, Seferovic M, Pace RM, Aagaard KM. The microbiome in preterm birth. Best Pract Res Clin Obstet Gynaecol 2018; 52: 103-113.

16. Lohmann P, Luna RA, Hollister EB, et al. The airway microbiome of intubated premature infants: characteristics and changes that predict the development of bronchopulmonary dysplasia. Pediatr Res 2014; 76: 294-301.

17. Marsland BJ, Trompette A, Gollwitzer ES. The gutlung axis in respiratory disease. Ann Am Thorac Soc 2015; 12(Suppl 2): S150-S156.

18. Ranucci G, Buccigrossi V, de Freitas MB, Guarino A, Giannattasio A. Early-life intestine microbiota and lung health in children. J Immunol Res 2017; 2017: 8450496.

19. Arrieta MC, Stiemsma LT, Dimitriu PA, et al. Early infancy microbial and metabolic alterations affect risk of childhood asthma. Sci Transl Med 2015; 7: 307 ra152.

20. Ipci K, Altintoprak N, Muluk NB, Senturk M, Cingi C. The possible mechanisms of the human microbiome in allergic diseases. Eur Arch Otorhinolaryngol 2017; 274: 617-626.
21. Varon E, Levy C, De La Rocque F, et al. Impact of antimicrobial therapy on nasopharyngeal carriage of Streptococcus pneumoniae, Haemophilus influenzae, and Branhamella catarrhalis in children with respiratory tract infections. Clin Infect Dis 2000; 31: 477-481.

22. Salter SJ, Turner C, Watthanaworawit W, et al. A longitudinal study of the infant nasopharyngeal microbiota: The effects of age, illness and antibiotic use in a cohort of South East Asian children. PLoS Negl Trop Dis 2017; 11: e0005975.

23. Budden KF, Gellatly SL, Wood DL, et al. Emerging pathogenic links between microbiota and the gutlung axis. Nat Rev Microbiol 2017; 15: 55-63.

24. Abrahamsson TR, Jakobsson HE, Andersson AF, Björksten B, Engstrand L, Jenmalm MC. Low gut microbiota diversity in early infancy precedes asthma at school age. Clin Exp Allergy 2014; 44: 842-850.

25. Warner BB, Hamvas A. Lungs, microbes and the developing neonate. Neonatology 2015; 107: 337 343.

26. Trompette A, Gollwitzer ES, Yadava K, et al. Gut microbiota metabolism of dietary fiber influences allergic airway disease and hematopoiesis. Nat Med 2014; 20: 159-166.

27. Chen Y, Blaser MJ. Helicobacter pylori colonization is inversely associated with childhood asthma. J Infect Dis 2008; 198: 553-560.

28. Lankarani KB, Honarvar B, Athari SS. The mechanisms underlying helicobacter pylori-mediated protection against allergic asthma. Tanaffos 2017; 16: 251-259.

29. Leyer GJ, Li S, Mubasher ME, Reifer C, Ouwehand AC Probiotic effects on cold and influenza-like symptom incidence and duration in children. Pediatrics 2009; 124: e172-e179.

30. Bruzzese E, Callegari ML, Raia V, et al. Disrupted intestinal microbiota and intestinal inflammation in children with cystic fibrosis and its restoration with Lactobacillus GG: a randomised clinical trial. PloS One 2014; 9: e87796.

31. Pammi M, Lal CV, Wagner BD, et al. Airway microbiome and development of bronchopulmonary dysplasia in preterm infants: a systematic review. J Pediatr 2019; 204: 126-133.e2.

32. van Waarde WM, Brus F, Okken A, Kimpen JL. Ureaplasma urealyticum colonization, prematurity and bronchopulmonary dysplasia. Eur Respir J 1997; 10: $886-890$

33. Wagner BD, Sontag MK, Harris JK, et al. Airway microbial community turnover differs by BPD severity in ventilated preterm infants. PloS One 2017; 12: e0170120. 
34. Ozdemir R, Erdeve O, Dizdar EA, et al. Clarithromycin in preventing bronchopulmonary dysplasia in Ureaplasma urealyticum-positive preterm infants. Pediatrics 2011; 128: e1496-e1501.

35. Hassan HE, Othman AA, Eddington ND, et al. Pharmacokinetics, safety, and biologic effects of azithromycin in extremely preterm infants at risk for ureaplasma colonization and bronchopulmonary dysplasia. J Clin Pharmacol 2011; 51: 1264-1275.

36. Imamura $\mathrm{T}$, Sato $\mathrm{M}$, Go $\mathrm{H}$, et al. The microbiome of the lower respiratory tract in premature infants with and without severe bronchopulmonary dysplasia. Am J Perinatol 2017; 34: 80-87.

37. Di Cicco M, Pistello M, Jacinto T, et al. Does lung microbiome play a causal or casual role in asthma? Pediatr Pulmonol 2018; 53: 1340-1345.

38. Costa AN, Costa FMD, Campos SV, Salles RK, Athanazio RA. The pulmonary microbiome: challenges of a new paradigm. J Bras Pneumol 2018: 44: 424-432.

39. Dickson RP, Erb-Downward JR, Martinez FJ, Huffnagle GB. The microbiome and the respiratory tract. Annu Rev Physiol 2016; 78: 481-504.

40. Perez GF, Perez-Losada M, Isaza N, Rose MC, ColbergPoley AM, Nino G. Nasopharyngeal microbiome in premature infants and stability during rhinovirus infection. J Investig Med 2017; 65: 984-990.

41. Bisgaard H, Hermansen MN, Buchvald F, et al. Childhood asthma after bacterial colonization of the airway in neonates. N Engl J Med 2007; 357: 14871495.

42. Teo SM, Mok D, Pham $\mathrm{K}$, et al. The infant nasopharyngeal microbiome impacts severity of lower respiratory infection and risk of asthma development. Cell Host Microbe 2015; 17: 704-715.

43. Bisgaard $\mathrm{H}$, Hermansen MN, Bonnelykke $\mathrm{K}$, et al. Association of bacteria and viruses with wheezy episodes in young children: Prospective birth cohort study. BMJ 2010; 341: c4978.

44. Ege MJ, Mayer M, Normand AC, et al; GABRIELA Transregio 22 Study Group. Exposure to environmental microorganisms and childhood asthma. N Engl J Med 2011; 364: 701-709.

45. Herbst T, Sichelstiel A, Schar C, et al. Dysregulation of allergic airway inflammation in the absence of microbial colonization. Am J Respir Crit Care Med 2011; 184: 198-205.

46. Stoltz DA, Meyerholz DK, Pezzulo AA, et al. Cystic fibrosis pigs develop lung disease and exhibit defective bacterial eradication at birth. Sci Transl Med 2010; 2: 29ra31.

47. Martin C, Burgel PR, Lepage P, et al. Host-microbe interactions in distal airways: relevance to chronic airway diseases. Eur Respir Rev 2015; 24: 78-91.
48. Frayman KB, Armstrong DS, Carzino R, et al. The lower airway microbiota in early cystic fibrosis lung disease: A longitudinal analysis. Thorax 2017; 72: 1104-1112.

49. Gangell C, Gard S, Douglas T, et al; AREST CF Inflammatory responses to individual microorganisms in the lungs of children with cystic fibrosis. Clin Infect Dis 2011; 53: 425-432.

50. Zemanick ET, Wagner BD, Robertson CE, et al. Assessment of airway microbiota and inflammation in cystic fibrosis using multiple sampling methods. Ann Am Thorac Soc 2015; 12: 221-229.

51. Prevaes SM, de Winter-de Groot KM, Janssens HM, et al. Development of the nasopharyngeal microbiota in infants with cystic fibrosis. Am J Respir Crit Care Med 2016; 193: 504-515.

52. Vissing $\mathrm{NH}$, Chawes $\mathrm{BL}$, Bisgaard $\mathrm{H}$. Increased risk of pneumonia and bronchiolitis after bacterial colonization of the airways as neonates. Am J Respir Crit Care Med 2013; 188: 1246-1252.

53. Sakwinska O, Bastic Schmid V, Berger B, et al Nasopharyngeal microbiota in healthy children and pneumonia patients. J Clin Microbiol 2014; 52: 15901594

54. Lu W, Yu J, Ai Q, Liu D, Song C, Li L. Increased constituent ratios of Klebsiella sp., Acinetobacter sp., and Streptococcus sp. and a decrease in microflora diversity may be indicators of ventilator-associated pneumonia: A prospective study in the respiratory tracts of neonates. PloS One 2014; 9: e87504.

55. Linares DM, Ross P, Stanton C. Beneficial microbes: The pharmacy in the gut. Bioengineered 2016; 7: 1120.

56. Forsythe P. Probiotics and lung immune responses Ann Am Thorac Soc 2014; 11 (Suppl 1): S33-S37.

57. Alexandre Y, Le Blay G, Boisramé-Gastrin S, et al Probiotics: A new way to fight bacterial pulmonary infections? Med Mal Infect 2014; 44: 9-17.

58. Bermon S, Petriz B, Kajeniene A, Prestes J, Castell L, Franco OL. The microbiota: an exercise immunology perspective. Exerc Immunol Rev 2015; 21: 70-79.

59. Banupriya B, Biswal N, Srinivasaraghavan R, Narayanan P, Mandal J. Probiotic prophylaxis to prevent ventilator associated pneumonia (VAP) in children on mechanical ventilation: an open-label randomized controlled trial. Intensive Care Med 2015; 41: 677-685.

60. Hojsak I, Snovak N, Abdović S, Szajewska H, Mišak Z, Kolaček S. Lactobacillus GG in the prevention of gastrointestinal and respiratory tract infections in children who attend day care centers: a randomized, double-blind, placebo-controlled trial. Clin Nutr 2010; 29: 312-316. 
61. Amaral MA, Guedes GHBF, Epifanio M, Wagner MB, Jones MH, Mattiello R. Network meta-analysis of probiotics to prevent respiratory infections in children and adolescents. Pediatr Pulmonol 2017; 52: 833-843.

62. Wang Y, Li X, Ge T, et al. Probiotics for prevention and treatment of respiratory tract infections in children: A systematic review and meta-analysis of randomized controlled trials. Medicine (Baltimore) 2016; 95: e4509.

63. Zhang J, Ma JY, Li QH, Su H, Sun X. Lactobacillus rhamnosus GG induced protective effect on allergic airway inflammation is associated with gut microbiota. Cell Immunol 2018; 332: 77-84.

64. Chen YS, Jan RL, Lin YL, Chen HH, Wang JY. Randomized placebo-controlled trial of lactobacillus on asthmatic children with allergic rhinitis. Pediatr Pulmonol 2010; 45: 1111-1120.

65. Wei X, Jiang $P$ Liu J Sun R, Zhu L. Association between probiotic supplementation and asthma incidence in infants: a meta-analysis of randomized controlled trials. J Asthma 2019; 1-12.

66. Anderson JL, Miles C, Tierney AC. Effect ofprobiotics on respiratory, gastrointestinal and nutritional outcomes in patients with cystic fibrosis: a systematic review. J Cyst Fibros 2017; 16: 186-197.

67. Ananthan A, Balasubramanian H, Rao S, Patole S. Probiotic supplementation in children with cystic fibrosis-a systematic review. Eur J Pediatr 2016; 175: $1255-1266$.

68. NikniazZ, Nikniaz L, Bilan N, Somi MH, Faramarzi E. Does probiotic supplementation affect pulmonary exacerbation and intestinal inflammation in cystic fibrosis: A systematic review of randomized clinical trials. World J Pediatr 2017; 13: 307-313.

69. Van Biervliet, S, Hauser B, Verhulst S, et al. Probiotics in cystic fibrosis patients: A double blind crossover placebo controlled study: Pilot study from the ESPGHAN Working Group on Pancreas/CF. Clin Nutr ESPEN 2018; 27: 59-65.
70. Bruzzese E, Raia V, Ruberto E, et al. Lack of efficacy of Lactobacillus GG in reducing pulmonary exacerbations and hospital admissions in children with cystic fibrosis: A randomised placebo controlled trial. J Cyst Fibros 2018; 17: 375-382.

71. Lu CY, Ni YH. Gut microbiota and the development of pediatric diseases. J Gastroenterol 2015; 50: 720726.

72. Ranucci G, Buccigrossi V, Borgia E, et al. Galactooligosaccharide/polidextrose enriched formula protects against respiratory infections in infants at high risk of atopy: a randomized clinical trial. Nutrients 2018; 10: E286.

73. Sierra C,Bernal MJ Blasco J, et al. Prebiotic effect during the first year of life in healthy infants fed formula containing GOS as the only prebiotic: A multicentre, randomised, double-blind and placebocontrolled trial. Eur J Nutr 2015; 54: 89-99.

74. Niele N, van Zwol A, Westerbeek EA, Lafeber HN, van Elburg RM. Effect of non-human neutral and acidic oligosaccharides on allergic and infectious diseases in preterm infants. Eur J Pediatr 2013; 172: 317-323.

75. Shahramian I, Kalvandi G, Javaherizadeh H, et al. The effects of prebiotic supplementation on weight gain, diarrhoea, constipation, fever and respiratory tract infections in the first year of life. J Paediatr Child Health 2018; 54: 875-880.

76. Klaenhammer TR, Kleerebezem M, Kopp MV Rescigno M. The impact of probiotics and prebiotics on the immune system. Nat Rev Immunol 2012; 12: 728-734.

77. Dickson RP, Erb-Downward JR, Huffnagle GB. The role of the bacterial microbiome in lung disease. Expert Rev Respir Med 2013; 7: 245-257. 